\title{
Relationship between peripheral blood eosinophil count and the severity and prognosis of children with Duchenne Muscular Dystrophy : a new potentially useful predictor?
}

\author{
Zhi Jiang \\ Hunan Children's Hospital \\ Hongmei Liao ( $\nabla$ hnliaohm006@sina.cn ) \\ Hunan Children's Hospital \\ Liwen Wu \\ Hunan Children's Hospital \\ Wenjing Hu \\ Hunan Children's Hospital \\ Liming Yang \\ Hunan Children's Hospital
}

\section{Bo Chen}

Hunan Children's Hospital

\section{Zeshu Ning}

Hunan Children's Hospital

Jingwen Tang

Hunan Children's Hospital

\section{Rong Xu}

Hunan Children's Hospital

Mei Chen

Hunan Children's Hospital

\section{Feng Guo}

Hunan Children's Hospital

Shulei Liu

Hunan Children's Hospital

\section{Research Article}

Keywords: eosinophils, Duchenne Muscular Dystrophy, inflammation, prognosis, curative effect

Posted Date: March 16th, 2022 
DOI: https://doi.org/10.21203/rs.3.rs-1383155/v2

License: (c) (1) This work is licensed under a Creative Commons Attribution 4.0 International License. Read Full License 


\section{Abstract}

Objective To investigate the effect of peripheral blood eosinophil count on the severity and prognosis of Duchenne Muscular Dystrophy凶DMD).

Method The clinical data of 145 patients with DMD and 150 normal healthy children in the same period were collected retrospectively. The results of the two groups were analysed and compared. Logistic regression and Cox regression analysis were used to explore the influence of eosinophils on DMD. The relationship between eosinophils and the long-term efficacy and prognosis of DMD were followed up.

Result Compared with the control group, the eosinophil count of children in DMD group was slightly lower $(Z=2.163, p=0.031)$. Eosino-phil count at visit and follow-up was slightly negatively correlated with muscle strength score to visit and follow-up, and the correlati-on coefficients were as follows: $\rho=-0.245 \mathbb{Z}$ $p=0.040 \rrbracket \rho=-0.137 \rrbracket p=0.032$. Eosinophils were the protective factor for high muscle strength score of visit, $O R=0.007,95 \% C /(0.001-0.276) \llbracket p=0.008, O R=0.038,95 \% C /(0.002-0.752), p=0.032$ after adjusting for age and other factors. Eosinophils were also the protective factor for elevated muscle stre-ngth score at follow-up visit, $(O R=0.012,95 \% C / 0.001-0.645, p=0.029)$, after adjusting for age and other factors $(O R=0.033,95 \% C / 0.001-0.121, p=0.039)$. Univariate analysis found that eosinophils affected the curative effect during follow-up visit, $H R=2.739,95 \% C /(1.294-5.979) p=0.008$. Further adjustment for age and other factors multivariate Cox regression analysis found that eosinophils affected the curative effect $(H R=1.127,95 \% C / 1.109-1.246, p=0.020 \rrbracket$.

Conclusion The eosinophil count in peripheral blood was related to the severity of DMD, and can predict the curative effect and prognosis to a certain extent. Eosinophil count may be a potentially useful predictor.

\section{Introduction}

Duchenne muscular dystrophy(DMD) is a rare fatal X-linked recessive hereditary myopathy[1,2]. The treatment of DMD is always a difficult problem to date. Dystrophin protein deletion or functional defect caused by gene abnormality, chronic inflammatory reaction and oxidative stress are its pathogenesis[3,4,5]. Immune and inflammatory processes downstream of dystrophin deficiency, as well as metabolic abnormalities, autophagy deficiency and loss of regeneration are the causes of muscle pathology of DMD[6]. Regulating inflammatory response and inducing immune tolerance to the expression of dystrophin are the key to the success of dystrophin replacement therapy[7].

Inflammatory response plays an important role in DMD. Chronic degeneration and regeneration of muscle fibers can achieve nuclear factor-KB囚NF-KB囚abnormal activation of inflammatory pathways leads to progressive membrane injury and eventually muscle degeneration[8]. The increase of proinflammatory factors and the inhibition of the inflammatory response contribute to improving the muscle strength of DMD. Some studies suggest that muscle enzymes in children with DMD with inflammatory level are elevated[3]. Inhibiting the level of inflammation in children with DMD is helpful to control the disease [9]. 
Is there a simple clinical indicator that can not only reflect the inflammatory response level of DMD, but also simply reflect the muscle strength state or prognosis of DMD? We hope to be able to find a simple and practical biomarker from the clinical point of view, which can quickly judge the muscle strong state and muscle injury degree of DMD, and reflect the prognosis to a certain extent.

Eosinophils are common inflammatory cells in peripheral blood, which is mainly related to allergy and parasitic infection. Literature has confirmed that there is eosinophil infiltration in DMD muscle tissue, and literature has confirmed that it can promote the repair of muscle cells [10]. Eosinophils have recently been considered as regulators of immune homeostasis, inhibiting the pro-inflammatory response of overreaction by secreting specific molecules that inhibit the immune response [11] .Peripheral blood eosinophils are associated with prognosis of some tumors, chronic obstructive pulmonary disease (COPD) and infectious diseases[12,13]. And peripheral blood eosinophils increase in some tumors has a relatively good prognosis[14]. Therefore, we explored the relationship between eosinophils in peripheral blood and the severity, curative effect or prognosis of $\mathrm{DMD}$, hoping to be able to find a difinite regularity.

\section{Method}

\section{1 study design}

Outpatient and inpatient children with DMD in Hunan children's Hospital from January 1, 2012 to December 31, 2020 were collected retrospectively.Inclusion criteria:1) Boys aged about 2-5 years (or slightly less than 2 years) with typical clinical symptoms.2)Genetic testing verified that was DMD. 3) They had not been dealt with in the time of the visit.

Exclusion criteria:1) DMD was precluded by gene test. 2)There were nothing more serious medical diseases such as infection, dia-rrhea and allergy, which could affect the examination results.3)The children did not complete follow-up and related examinations as werequired.4) Withdrawal or loss of follow-up due to other reasons.150 normal healthy children in the same period were perceived as thecontrol group. Inclusion criteria:1)Boys were healthy and without o-bvious diseases within 3 months for follow up.2)They were prepari-ng for vaccination or physical examination in our hospital.3) They were normal healthy volunteers participating in the normal reference value plan for blood and other indicators of normal children in o-ur hospital and Beijing Children's Hospital(after physical examinatio-n,etc.).

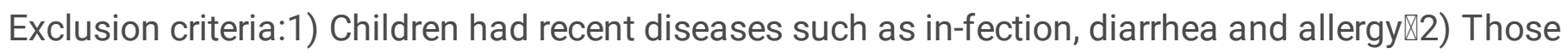
children who have other de-velopmental abnormalities or other metabolic abnormalities. 3) Therewere other diseases that may influence the results. 4) Exit for otherreasons. This study was approved by the ethics committee of Hunanchildren's hospital.

\section{2 collection of case data}

The clinical data of all selected cases was collected retrospectively, including age, and muscle strength scores. All laboratory data such as blood routine, myocardial enzyme spectrum,etc were collected, but 
vitamin D3\at the time of visit, $n=55$ \at follow-up visit $n=46$ and C-reactive protein (CRP) $₫ A$ the time of visit $\ n=62 \bigotimes$ at follow-up visit $\ n=41$ \levels were collected in some children.All blood routine and biochemical tests were at a.m.8: 00 or so.

\section{3 treatment}

All children with DMD choose the treatment plan according to the condition of the children and the informed consent of their parents. 1) Conservative treatment: After learning that the disease was incurable at present, some parents choose to give up regular prednisone treatment and only gave conservative treatment such as fructose sodium diphosphate and coenzyme Q10. A few parents of children had not decided how to give conservative treatment during the treatment period, but they were followed up regularly. 2)Prednisone therapy (Children started treatment around the age of 5, and some may be slightly delayed.), and the side effects were monitored regularly. Prednisone's dose was $0.75 \mathrm{mg} / \mathrm{d}, \mathrm{QD}$. Children were routinely supplemented with calcium and vitamin D.

\subsection{Folow up}

The patients were followed up regularly after discharging or leaving the hospital, and returned to the outpatient clinic every 3-6 months to evaluate muscle strength and development. The follow-up was terminated on April 30, 2021. The efficacy at the last follow-up visit was taken as the termination event (Invalid as the end event, i.e. the muscle strength score increased by 1 point or more, and other cases were censored events).

\section{5 statistical treatment}

If all data were normally distributed, they were expressed as mean \pm standard deviation. If the distribution was skewed, it was expressed as the median $(P 25, P 75)$. For normal distribution data, independent sample t-test or paired t-test was used for mean comparison ( $\mathrm{t}$ 'test is used for uneven variance), and independent sample Mann-Whitney $U$ test was used for non normal distribution. Pearson correlation analysis was used for normal distribution data, and Spearman correlation analysis was used for non normal distribution data. Logistic regression was used to evaluate the risk factors, Kaplan-Meier analysis and Cox regression analysis were used to explore the impact of various factors on the treatment effect. All data were processed using SPSS 24.0. P $<0.05$ was statistically significant.

\section{Results}

\subsection{Comparison of general data between children with DMD and normal control group}


A total of 287 children entered this study, except that 80 cases had not completed blood routine and muscle enzyme tests as required, 40 cases lost the follow up because they did not follow-up on time or change their contact information, 22 cases withdrew because of pulmonary infection and diarrhea during treatment or follow up, and the remaining 145 children completed the study. 150 children in the control group were healthy children in the same period. The level of eosinophils was 0 at $0-0.1 \times 10^{9} / I$, and greater than or equal to $0.1 \times 10^{9} /$ I was 1 . The results of gene testing showed that 145 cases completed gene testing, including 92 cases of gene deletion (80 cases of large fragments and 12 cases of small fragments), 22 cases of gene duplication and variation (4 cases of single exon duplication, 16 cases of multi exon duplication) and 31 cases of point mutation (4 missense variants, 16 nonsense variants, 6 frameshift variants, 5 splice variants). The mean follow up time of DMD patients was $1.67 \rrbracket 0.50,3.25 \rrbracket$ years. The comparison of age, blood routine and biochemical results between the two groups is shown in Table 1.

Table 1 Comparison of baseline data between patients with DMD and normal control group 


\begin{tabular}{|c|c|c|c|c|}
\hline & & Control group & Value & $P$ \\
\hline & $N=145$ & $N=150$ & & \\
\hline Age & $3.83 \rrbracket 1.42,6.08 \rrbracket$ & $2.92 \rrbracket 1.17,5.92 \rrbracket$ & -0.795 & 0.427 \\
\hline Total protein & $66.18 \pm 4.13$ & $68.66 \pm 4.41$ & 4.820 & 0.000 \\
\hline Albumin & $42.52 \pm 3.71$ & $46.74 \pm 2.39$ & 11.042 & 0.000 \\
\hline Globulin & $23.67 \pm 3.87$ & $21.93 \pm 3.02$ & 4.193 & 0.000 \\
\hline Urea nitrogen & $3.82 \pm 1.31$ & $4.58 \pm 1.07$ & 4.463 & 0.000 \\
\hline White blood cell & 8.00ه6.39ه9.82》 & $7.55 \otimes 6.40 \otimes 9.41 \rrbracket$ & 0.880 & 0.379 \\
\hline Neutrophils & $3.43 \rrbracket 2.52 \rrbracket 4.36 \rrbracket$ & $2.87 \rrbracket 2.30 \rrbracket 3.86 \rrbracket$ & 2.723 & 0.006 \\
\hline Lymphocyte & $3.34 \llbracket 2.69 \rrbracket 4.03 \rrbracket$ & $3.47 \rrbracket 2.87 \rrbracket 4.73 \rrbracket$ & -1.407 & 0.159 \\
\hline Monocyte & $0.35 \rrbracket 0.27 \rrbracket 0.49 \rrbracket$ & $0.41 \rrbracket 0.32 \llbracket 0.59 \rrbracket$ & -2.896 & 0.004 \\
\hline Red blood cell & $4.65 \pm 0.37$ & $4.79 \pm 0.39$ & 3.060 & 0.002 \\
\hline Hemoglobin & $122.83 \pm 10.29$ & $128.51 \pm 9.89$ & 4.651 & 0.000 \\
\hline Eosinophils & $0.16 \rrbracket 0.09 \rrbracket 0.29 \rrbracket$ & $0.21 \rrbracket 0.12 \llbracket 0.32 \rrbracket$ & 2.163 & 0.031 \\
\hline Basophil & $0.01 \rrbracket 0.003 \rrbracket 0.02 \rrbracket$ & $0.01 \rrbracket 0.002 \rrbracket 0.03 \rrbracket$ & 0.940 & 0.347 \\
\hline Platelet & $311.24 \pm 92.23$ & $308.32 \pm 65.48$ & 0.297 & 0.767 \\
\hline Vitamin D3 & $47.91 \pm 11.48$ & $82.60 \pm 23.12$ & 6.439 & 0.000 \\
\hline Creatinine & $18.41 \pm 7.79$ & $35.25 \pm 10.18$ & 13.375 & 0.000 \\
\hline Proportion of eosinophils & $0.02 \rrbracket 0.01,0.03 \rrbracket$ & $0.03 \rrbracket 0.02,0.05 \rrbracket$ & 3.454 & 0.001 \\
\hline
\end{tabular}

A total of 145 children with DMD completed this follow up study. Compared with the first visit, the eosinophil count, erythrocyte, hemoglobin and uric acid囚etc $\rrbracket$ at the follow-up visit were statistically significant $(P<0.05$ or 0.01$)$. The comparison of baseline data between the initial visit and the follow-up visit is shown in Table 2.

Table 2 Comparison of data between the first visit and follow-up visit of children with DMD 


\begin{tabular}{|c|c|c|c|c|}
\hline & \multirow{3}{*}{$\begin{array}{l}\text { At visit } \\
\mathrm{N}=145\end{array}$} & Follow & \multirow[t]{3}{*}{ Value } & \multirow[t]{3}{*}{$P$} \\
\hline & & Up & & \\
\hline & & $N=145$ & & \\
\hline Age & $3.83 \rrbracket 1.42 \rrbracket 6.08 \rrbracket$ & $5.25 \llbracket 3.16 \rrbracket 7.16 \rrbracket$ & 10.449 & 0.000 \\
\hline Total bilirubin & $7.89 \llbracket 6.00 \rrbracket 10.90 \rrbracket$ & $7.92 \llbracket 6.30 \rrbracket 10.49 \rrbracket$ & -0.484 & 0.629 \\
\hline Direct bilirubin & $2.70 \rrbracket 1.90 \rrbracket 3.66 \rrbracket$ & $2.33 \rrbracket 1.60 \rrbracket 3.20 \rrbracket$ & 1.733 & 0.083 \\
\hline Indirect bilirubin & $5.30 \rrbracket 3.80 \rrbracket 7.58 \rrbracket$ & $5.70 \rrbracket 4.20 \rrbracket 7.30 \rrbracket$ & 0.566 & 0.577 \\
\hline Total protein & $66.18 \pm 4.13$ & $66.24 \pm 4.29$ & 0.425 & 0.672 \\
\hline Albumin & $42.52 \pm 3.71$ & $42.62 \pm 3.35$ & 0.049 & 0.961 \\
\hline Globulin & $23.67 \pm 3.87$ & $23.62 \pm 2.97$ & 0.398 & 0.692 \\
\hline Creatinine & $18.41 \pm 7.79$ & $23 \llbracket 41 \pm 9.17$ & 2.933 & 0.006 \\
\hline Urea nitrogen & $3.82 \pm 1.31$ & $3.68 \pm 0.98$ & 0.233 & 0.817 \\
\hline Uric acid & $216.59 \pm 71.59$ & $249.46 \pm 58.45$ & 2.245 & 0.035 \\
\hline Total bile acid & $4.40 \rrbracket 3.00 \rrbracket 8.07 \rrbracket$ & $3.40 \rrbracket 2.20 \rrbracket 4.87 \rrbracket$ & -2.867 & 0.004 \\
\hline CK & $\begin{array}{l}4177.00 \rrbracket 1698.70 \rrbracket \\
9831.30 \rrbracket\end{array}$ & $\begin{array}{l}7108.30 \rrbracket 1993.2 \rrbracket \\
11755.30 \rrbracket\end{array}$ & 2.074 & 0.038 \\
\hline CK-MB & $188.8 \rrbracket 109.76 \rrbracket 298.00 \rrbracket$ & $184.09 \rrbracket 119.35 \llbracket 284.58 \rrbracket$ & 1.441 & 0.149 \\
\hline LDH & $1203.6(790.00,1322.00)$ & $\begin{array}{l}994.00 \rrbracket 760.00 \rrbracket \\
1262.60 \rrbracket\end{array}$ & -1.039 & 0.229 \\
\hline $\begin{array}{l}\text { Alanine } \\
\text { aminotransferase }\end{array}$ & $322.70 \pm 181.07$ & $303.75 \pm 140.55$ & 1.240 & 0.218 \\
\hline $\begin{array}{l}\text { Aspartate } \\
\text { aminotransferase }\end{array}$ & $244.2 \rrbracket 139.60 \rrbracket 315.00 \rrbracket$ & 195.90冈146.90囚292.70》 & 2.001 & 0.045 \\
\hline Eosinophils & $0.16 \varangle 0.09 \rrbracket 0.29 \rrbracket$ & $0.11 \rrbracket 0.07 \rrbracket 0.19 \rrbracket$ & 2.251 & 0.032 \\
\hline Basophil & $0.01 \rrbracket 0.003 \rrbracket 0.02 \rrbracket$ & $0.01 \rrbracket 0.01 \rrbracket 0.02 \rrbracket$ & 0.766 & 0.444 \\
\hline Red blood cell & $4.65 \pm 0.37$ & $4.85 \pm 0.42$ & 4.577 & 0.000 \\
\hline Hemoglobin & $122.83 \pm 10.29$ & $129.99 \pm 12.50$ & 5.521 & 0.000 \\
\hline CRP & $0.75 \llbracket 0.18 \rrbracket 1.21 \rrbracket$ & $0.50 \rrbracket 0.30 \rrbracket 0.84 \rrbracket$ & 1.521 & 0.128 \\
\hline Platelet & $311.24 \pm 92.23$ & $299.76 \pm 66.14$ & 1.079 & 0.284 \\
\hline Vitamin D3 & $47.91 \pm 11.48$ & $45.22 \pm 9.23$ & 1.155 & 0.307 \\
\hline Vignos & $2 \otimes 1,3 \rrbracket$ & $2 \otimes 1,3 \rrbracket$ & 0.780 & 0.435 \\
\hline
\end{tabular}


scale

\begin{tabular}{|c|c|c|c|c|}
\hline Neutrophils & 3.43区2.52,4.36ه & $3.19 \rrbracket 2.71,4.41 \rrbracket$ & 0.895 & 0.371 \\
\hline Lymphocyte & 3.34ه2.69,4.03区 & $3.51 \rrbracket 2.51,4.27 \rrbracket$ & 0.330 & 0.741 \\
\hline Monocyte & $0.35 \varangle 0.27,0.49 \rrbracket$ & $0.40 \otimes 0.33,0.52 \rrbracket$ & -1.108 & 0.268 \\
\hline $\begin{array}{l}\text { Proportion of } \\
\text { eosinophils }\end{array}$ & $0.02 \varangle 0.01,0.03 \rrbracket$ & $0.02 \varangle 0.01,0.03 \rrbracket$ & 1.105 & 0.269 \\
\hline
\end{tabular}

\subsection{The effect of eosinophils on muscle strength score}

The correlation coefficient between eosinophils and vitamin D3 in the normal control group was $\rho=$ $0.299 \rrbracket p=0.012$. The correlation coefficient between eosinophils and vitamin D3 in DMD group was $\rho=$ $0.563 \rrbracket p=0.001$. The correlation coefficient between eosinophils and vitamin D3 was $\rho=0.267 \rrbracket p=0.207$.

Correlation coefficient between eosinophils and CRP at visit $\rho=-0.271 \rrbracket p=0.046$,eosinophils and CRP at follow-up visit $\rho=-0.189 \rrbracket p=0.035$.

Correlation coefficient between age and visit score was $\rho=0.528, p=0.000$, correlation coefficient between follow-up age and follow-up score was $\rho=0.512, p=0.000$,that was Statistically significant, The partial correlation analysis of controlling age found that the partial correlation coefficient between eosinophil count and visit score at visit was $-0.155, p=0.047$, and the partial correlation analysis at follow-up visit was $0.082, p=0.489$.

Correlation coefficient between eosinophils to visit and score of visit was $\rho=-0.245, p=0.040$. Correlation coefficient with lymphocytes was $\rho=0.257, p=0.004$, correlation coefficient with monocytes was $\rho=-0.195$, $p=0.032$. These were statistically significant.

Correlation coefficient between follow up visit eosinophils and fo-llow-up scores was $\rho=-0.137, p=0.032$. There was statistically signif-icant.Correlationcoefficient between eosinophils with lymphocytes at followup visit was $\rho=0.038, p=0.716$. Correlation coefficient betweeneosinophils and monocytes at follow-up visit was $\rho=0.46, p=0.656$. T-here was no statistical significance. Correlation coefficient between eosinophils and lymphocytes to the first visit was $\rho=0.244, p=0.007$, monocyte correlation coefficient was $\rho=0.182, p=0.044$.

\subsection{Effects of different treatment methods on eosinophil count}

There were 95 children in the prednisone treatment group. The number of eosinophils was 0.15 $(0.08,0.25)$ at the time of treatment and $0.08(0.05,0.17)$ at the time of follow-up. The comparison before and after treatment was $Z=3.157, p=0.002$. The correlation coefficient between the follow-up score and 
follow-up eosinophils was $\rho=-0.092, p=0.573$, and the correlation coefficient between visit score and eosinophils was $\rho=-0.259, p=0.041$.

There were 50 children in the conservative treatment group.The number of eosinophils in the conservative treatment group was $0.19(0.10,0.31)$ at the time of visit and $0.20(0.11,0.27)$ at the time of follow-up. The comparison before and after treatment was $Z=0.270, p=0.787$. The correlation coefficient between the follow-up score and eosinophils was $\rho=-0.233, p=0.004$, and the correlation coefficient between visit score and eosinophils was $\rho=-0.212, p=0.043$. Comparison of eosinophils in the conservative treatment group and prednisone treatment group before treatment, $Z=1.464, p=0.143$, There was no statistical difference. There was statistical difference after treatment, $Z=4.559, p=0.000$.

\subsection{Eosinophils affected muscle strength score of DMD}

Single factor regression analysis of DMD muscle strength score revealed that the age of visit $(O R=1.453,95 \% C / 1.180-1.789 . p=0.000)$,creatinine ( $O R=0.865,95 \% C / 0.754-0.953 . p=0.039)$ lymphocyte $(O R=0.646,95 \% C 10.434-0.960 p=0.031)$,mononuclear $(O R=0.046,95 \% C / 0.003-0.849 . p=0.038)$ \eosinophils $(O R=0.007,95 \% C / 0.001-0.276 . p=0.008)$ were statistically significant. Age was a risk factor and others were protective factors for muscle strength score.

In the multivariate regression controlling for age and other factors, it was found that eosinophils $(O R=0.038,95 \% \mathrm{Cl} 0.002-0.752$. $p=0.032)$ were protective factors of muscle strength score. The higher the eosinophils, the lower the score, the higher the muscle strength. The results of multivariate regression of influencing factors of muscle strength at the first visit are shown in Table 3.

Table 3 Multivariate regression analysis of muscle strength scores in children with DMD

\begin{tabular}{|lllllll|}
\hline & $\mathrm{B}$ & $\mathrm{SE}$ & Wald & $\operatorname{Exp~B}$ & $95 \% \mathrm{Cl}$ & $\mathrm{P}$ \\
\hline Age & 0.962 & 0.331 & 8.474 & 2.619 & $1.369-5.003$ & 0.004 \\
\hline Lymphocyte & -0.633 & 0.412 & 2.362 & 0.531 & $0.237-1.190$ & 0.124 \\
\hline Eosinophils & -3.324 & 1.551 & 4.590 & 0.038 & $0.002-0.752$ & 0.032 \\
\hline Constant & 1.094 & 0.893 & 5.991 & 2.983 & & 0.672 \\
\hline
\end{tabular}

Using univariate regression of follow-up score we found the age of follow-up $(O R=1.334,95 \% C / 1.134$ $1.570, p=0.000)$, treatment $(O R=0.404,95 \% C / 0.178-0.918 . p=0.030)$,lymphocytes $(O R=0.630,95 \% C 10.439$ 0.904. $p=0.012)$, monocytes $(O R=0.015,95 \% C / 0.001-0.405 . p=0.013)$,Visit score $(O R=11.543,95 \% C / 5.607-$ 26.297. $p=0.000)$. Following up eosin-ophils $(O R=0.012,95 \% C / 0.001-0.645 . p=0.029)$,Eosinophils at visit $(O R=0.016,95 \% C / 0.002-0.586, p=0.024)$ were statistically significant.

Further control of age and other factors was discovered by multivariate regression, treatment $(O R=0.167,95 \% C 10.030-0.931, p=0.041)$,Visit score $(O R=13.582,95 \% C / 1.332-138.492$. 
$p=0.028)$, The effect of follow-up eosinophils ( $O R=0.033,95 \% C / 0.001-0.821, p=0.039)$ on muscle strength score was statistically significant.The results of multivari-ate regression of muscle strength influencingfactors during follow-up are shown in Table 4.

Table 4 Multivariate regression table of DMD follow-up muscle strength score

\begin{tabular}{|lllllll|}
\hline & $\mathrm{B}$ & $\mathrm{SE}$ & Wald & $\operatorname{Exp~B}$ & $95 \% \mathrm{Cl}$ & $P$ \\
\hline Follow up age & 1.042 & 0.626 & 2.768 & 2.834 & $0.831-9.668$ & 0.096 \\
\hline Treatment & -1.788 & 0.876 & 4.167 & 0.167 & $0.030-0.931$ & 0.041 \\
\hline Score at visit & 2.609 & 1.185 & 4.849 & 13.582 & $1.332-138.492$ & 0.028 \\
\hline Follow up eosinophils & -5.721 & $2 \varangle 764$ & 4.283 & 0.033 & $0.001-0.821$ & 0.039 \\
\hline Constant & -3.738 & 1.583 & 5.577 & 0.024 & & 0.018 \\
\hline
\end{tabular}

\subsection{Analysis of the effect of eosinophils on the efficacy of DMD}

Using univariate Cox regression analysis we found that the age of visit $(H R=0.851,95 \% \mathrm{Cl} 0.771$ $0.939, p=0.001)$, treatment $(H R=3.362,95 \% C / 1.222-5.607, p=0.000)$ and visit score $(H R=0.637,95 \% \mathrm{Cl}$ 0.454-0.863. $p=0.002)$,Lymphocytes $(H R=1.056,95 \% C h .003-$

$1.111, p=0.037)$, Granulocytes $(H R=0.785,95 \% C / 0.457-0.954, p=0.048)$ follow up gra-nulocytes $(H R=0.481,95 \% C / 0.254-0.912$. $p=0.025)$, Albumin ( $H R=1.131,95 \% C / 1.060-1.206 . p=0.002)$,Follow up eosinophils $(H R=2.739,95 \% C / 1.294-5.979 . p=0.008)$ there were statistically significant.

After further analysis of other factors, multivariate regression we found age at visit ( $H R=0.827,95 \%$ C10.701-0.974. $p=0.023)$,visit sco-re $(H R=0.354,95 \% C / 0.465-0.942 . p=0.008)$,treatment $(H R=7.596,95 \%$ $C / 2.138-26.990, p=0.002)$,eosinophils ( $H R=1.127,95 \% C / 1.109-1.246, p=0.020)$, these were statistically significant. It suggests that the higherthe eosinophils, the better the curative effect. Multivariate Cox regression results affecting the efficacy of DMD in children are shown in Table 5.

Table 5 Cox multivariate regression table of effective treatment in children with DMD

\begin{tabular}{|lllllll|}
\hline & $\mathrm{B}$ & $\mathrm{SE}$ & Wald & $\mathrm{HR}$ & $95 \% \mathrm{Cl}$ & $P$ \\
\hline Follow up eosinophils & 0.119 & 0.051 & 5.399 & 1.127 & $1.109-1.246$ & 0.020 \\
\hline Visit score & -0.471 & 0.156 & 7.006 & 0.354 & $0.465-0.942$ & 0.008 \\
\hline Treatment & 2.208 & 0.647 & 9.825 & 7.596 & $2.138-26.990$ & 0.002 \\
\hline Age of visit & -0.191 & 0.084 & 5.176 & 0.827 & $0.701-0.974$ & 0.023 \\
\hline
\end{tabular}


Kaplan-Meier analysis of different levels of eosinophils at follow-up showed that there were significant differences in the effective maintenance time of different levels of eosinophils for the treatment of DMD, see Fig. 1. The higher the level of eosinophils, the longer the effective maintenance time of treatment. There are also significant differences in the effective maintenance time of DMD treatment by different treatment methods during follow-up visit, see Fig. 2 The curative effect of prednisone treatment is significantly better than that of conservative treatment.

In Cox regression, it was found that there were also significant differences in the efficacy of different levels of eosinophils on DMD, see Fig. 3 The higher the level of eosinophils, the better the curative effect.

\section{Discussion}

A considerable amount of evidence shows that the inflammatory response has an influence on DMD.The continuous inflammatory response aggravates the disease severity of DMD [3,9]. Chronic inflammatory response is also the pathological feature of DMD muscle disease. These patients were accompanied by elevated levels of inflammatory factors such as interleukin-6(IL-6) and interferon- $\gamma($ IFN- $\gamma)$, tumor necrosis factor- $a(T N F-a)$,etc. There is also strong evidence that anti-inflammatory treatments such as prednisone therapy are effective. How to use a simple and effective marker to evaluate the level of inflammatory response in children with $\mathrm{DMD}$ is also a meaningful thing.

There is a chronic inflammatory response in children with DMD, and eosinophils are also engaged in the immune inflammatory response, although they are not the mainstream. Our partial correlation analysis found that there was a certain correlation between eosinophils and muscle strength score, which also confirmed that eosinophils had a certain effect on muscle strength.Our study confirmed that the level of eosinophils in children with DMD was negatively correlated with blood CRP. So it was negatively correlated with the level of inflammation in vivo. Therefore, the level of peripheral blood eosinophils was also negatively correlated with systemic inflammatory response, which apears to be related to the downregulation of inflammatory response. Compared with the case group, the level of eosinophils in the normal control group was slightly higher, and the difference was statistically significant.

Why? It is widely believed that eosinophils are a kind of infl-ammatory cells, which are related to allergy and parasitic infection. Its increase represents an inflammatory response. Why is it negatively correlated with CRP? A COPD study also confirmed that the e-osinophil count in peripheral blood was negatively correlated with CRP. The explanation of this article is that eosinophils enter the l-ung tissue when the inflammatory reaction is obvious, so the eosin-ophils in blood decreased [15]. Therefore, we speculate that there is a similar reason. It may be that eosinophils enter the muscle tissue,so the eosinophils in peripheral blood of DMD group are slightly lower than those of normal control group, and the proportion of eosinophils is the same.ls it related to the inhibition of the inflam-matory response by eosinophils? Eosinophils can maintain Th1/Th2 $2 \mathrm{~T}$ helper1/T helper2 $\varangle$ immune balance and inhibit excessive inflammatory response in the body[16].Therefore, we believe that eosi-nophils can inhibit the excessive 
inflammatory response in children with DMD. However, we still don't know what the cause is, whichneeds to be further studied.

Correlation analysis with motor score showed that the higher the eosinophilia, the lower the Vignos score, and the better the motor function. The lower the eosinophils, the worse the curative effect, as showed in the figure 1. However, it should be noted that the literature confirms that the number of eosinophils in peripheral blood is not related to the number of eosinophils in DMD muscle [17]. Therefore, this did not represent the number of eosinophils in muscle. The relationship between its change and the disease can not be compared with the relationship between the number of eosinophils in muscle and the disease.

Using univariate logistic regression we found that eosinophils were the protective factors with high motor function score whether at the time of visit or follow-up visit. This result was also supported after eliminating factors such as age, and drugs. After treatment, it was found that the lower the eosinophils in the prednisone treatment group, the more poor the curative effect. See the results of univariate KaplanMeier analysis, and the multivariate Cox regression also supports this conclusion.

The effect of eosinophils on the efficacy of DMD may come from its protective effect on muscle strength. Our study found that eosinophils were negatively correlated with exercise score before treatment, that is, positively correlated with muscle strength, and this correlation disappeared after prednisone treatment. However, Kaplan-Meier analysis and multivariate Cox analysis can still find this effect. At the time of follow-up, this correlation still existed in the conservative treatment group. It shows that eosinophils have a certain protective effect on DMD muscle strength. As for its mechanism, it is not very clear at present and needs to be further studied. Recent animal experiments have confirmed that eosinophils do not mediate the acute muscle injury of DMD[18], and it is unclear whether this is the case in humans. Therefore, eosinophils play a certain role in DMD muscle repair [10].But we also need further evidence.

In addition to regulating calcium and phosphorus metabolism and preventing osteoporosis, vitamin D3 also has immunomodulatory effect. It can down regulate the inflammatory response, which is of certain significance in multiple sclerosis and Systemic lupus erythematous(SLE) $[19,20]$. Vitamin D3 can down regulate the inflammatory response through the vitamin D receptor, enhance Treg ( $T$ regulatory) cell function and induce immune tolerance. Our study confirmed that the level of vitamin D3 in DMD group was significantly lower than that in normal control group, so its ability to down regulate the inflammatory response was significantly lower than that in normal control group.Our study confirmed that eosinophils are positively correlated with vitamin D. Whether they have the same ability to down regulate inflammatory response as vitamin $D$ remains to be further studied. The higher correlation between eosinophils and vitamin D in DMD group is due to the lower vitamin D level and lower eosinophils in DMD group than that in normal control group.

Eosinophils are closely related to lymphocytes. Our study confirmed that they slightly relate to lymphocytes. Eosinophils need lymphocyte activation. T lymphocytes are involved in the inflammatory response of DMD. Early studies confirmed that T cells decreased after prednisone treatment in patients with DMD, accompanied by reduced muscle necrosis and fibrosis. Muscle lymphocytes in patients with 
DMD. Our study confirmed that peripheral blood lymphocytes were the protective factor(univariate) of muscle strength score at the time of visit. Unfortunately, there was no significant statistical significance in further multivariate regression. It may be due to the insufficient number of cases or other interfering factors, or because the blood routine of follow-up visit was not a fixed time (The proportion of lymphocytes in children of different ages was different.), which could not be compared. All of these needs to be further studied. Studies have confirmed that the number of CD8/CD26 T cells in peripheral blood of patients with DMD is positively correlated with quantitative muscle score (QMT) (the higher the number, the higher the muscle strength), which is consistent with our results [21]. Studies have reported that CD49d can be used as a marker and potential therapeutic target of DMD disease process [22].

Eosinophils are also activated by monocytes, which participate in the inflammatory response of DMD. Monocyte macrophages appear in the early stage of muscle injury, in which M1区Monocyte 1区 mainly mediates the inflammatory response, and M2 $\triangle$ Monocyte $2 \triangle$ inhibits this response to repair muscle cells[23]. Animal experiments have confirmed that inflammatory monocytes improve the prognosis of DMD and may play a role through Chemokine receptor-2(CCR2) [24].

For DMD, the deletion of dystrophin protein is caused by genetic reasons. With the increase of age, muscle damage and symptoms, DMD generally loses motor function at about 12 years old (if untreated). The influence of age on sport score is obvious. With the increase of age, the vignos score gradually increases. Our research also supports this. Our Cox regression also found that the effective rate of treatment was getting lower with the increasing of age. With the increase of age, the interaction between the chronic activation of innate immunity and the degradation and regeneration of asynchrony and proximity produced an uncoordinated repair response, and this repair response itself may promote the development of the disease. With the increase of age, this trend is more obvious.

At present, more effective treatment of DMD is prednisone, but the adverse reactions of long-term treatment are also obvious. Relatively speaking, difecolate is more effective than prednisone (our patients did not choose this case because the number of using this drug was too small) [25]. Although the mechanism of prednisone treatment of DMD is not particularly clear $[26,27]$. Our study confirmed that prednisone can control the inflammatory response.Logsitic regression suggests that prednisone has a protective effect on muscle strength score, that is, muscle strength can be improved with prednisone treatment. Further Cox regression further confirmed that prednisone was effective, which was consistent with the literature report [28].

The deficiency of this study was that the number of cases was not much, the follow-up data were not enough, and the 6-minute walk test was not completed (It was a retrospective study \some children did not cooperate). The follow-up time of DMD children was not long enough. The data were all retrospective,many factors cannot be intervened, and it was more meaningful to have prospective study data. In this study, the number of muscle eosinophil and cytokine level on DMD was not further discussed in detail and in-depth at a higher level. In addition, this study did not involve specific mechanisms. 
Another disadvantage was that the detected eosinophils in peripheral blood cannot represent the infiltration level of eosinophis in DMD muscle,and the latter may be more valuable.

In conclusion, eosinophils in peripheral blood of children with DMD can reflect muscle strength score and peripheral blood inflammatory response,which may be related to the curative effect, clinically significant and reflect the prognosis to a certain extent. It may be clinically possible to preliminaries evaluate the condition and predict the curative effect by eosinophils count.

\section{Abbreviations}

ORCID

Open Researcher and Contributor Identity Document

DMD

Duchenne Muscular Dystrophy

OR

odds ratio

$\mathrm{Cl}$

confidence interval

NF-KB

nuclear factor-KB

COPD

chronic obstructive pulmonary disease

CRP

C-reactive protein

QD

quaque die

CK

creatine kinase

CK-MB

creatine kinase-MB

$\mathrm{LDH}$

Lactic dehydrogenase

IL-6

interleukin-6

IFN-Y

interferon-y:interferon- $\gamma$

TNF-a

tumor necrosis factor-a

Th1

T helper1

Th2 
T helper 2

SLE

Systemic lupus erythematous

Treg

$T$ regulatory

CD8

Cluster of differentiation 8

CD26

Cluster of differentiation 26

QMT

quantitative muscle score

CD49d

Cluster of differentiation 49d

M1

Monocyte 1

M2

Monocyte 2

CCR2

Chemokine receptor-2.

\section{Declarations}

\section{Acknowledgements}

The authors thank and acknowledge all of the participants who were enrolled in this Study.

\section{Authors' contributions}

ZJ wrote design research, extracted and analyzed data, completed

the text of main manuscripts and prepared figures. HML completed the revision of the paper and data collection. LWW directed the design of the research scheme.WJH completed the table design a-nd data review.LMY and BC completed data collection a-nd review.ZSN, JWT, XR, CM and F G have completed the revision and translation of papers. SLL completed the statistical processing of the data. All the authors reviewed and approved the manuscript.

\section{Funding}

This article did not receive any funding.

\section{Availability of data and materials}


The data that support the fndings of this work are available from the corresponding author upon reasonable request.

\section{Ethics approval and consent to participate}

This study was approved by the ethics committee of Hunan children's hospital.The treatment plan of DMD children obtained the informed consent of parents.

\section{Consent for publication}

Not applicable

\section{Competing interests}

No potential conflict of interest was reported by the author(s).

\section{Author details}

Department of Neurology, Hunan Children's Hospital, Changsha 410007, China.

\section{References}

1. Terrill JR, Duong MN, Turner R, Le Guiner C, Boyatzis A, Kettle AJ,et al.Levers of inflammation and oxidative stress, and a role for taurine in dystropathology of the golden retriever muscular dystrophy dog model for Duchenne muscular dystrophy. Redox Biol.2016:9:276-286.doi: 10.1016/j.redox.2016.08.016.

2. Finanger E, Vandenborne K, Finkel RS, Lee Sweeney H, Tennek-oon G,Yum S,et al. Phase 1 Study of Edasalonexent (CAT-1004), anOral NF-kappaB Inhibitor, in Pediatric Patients with Duchenne Mus-cular Dystrophy. J Neuromuscul Dis, 6(1), 43-54. doi:10.3233/JND-180341.

3. Cruz-Guzmán Odel R, Rodríguez-Cruz M, Escobar Cedillo RE.S-ystemic Inflammation in Duchenne Muscular Dystrophy: Associationwith Muscle Function and Nutritional Status. Biomed Res Int, 2015,891972. doi:10.1155/2015/891972.

4. Lecompte S,Abou-Samra M, Boursereau R, Noel L, Brichard SM. Skeletal muscle secretome in Duchenne muscular dystrophy: a pivotal anti-inflammatory role of adiponectin. Cell Mol Life Sci, 74(13), 2487-2501. doi:10.1007/s00018-017-2465-5.

5. Farini AđSitzia C, Cassani B, Cassinelli L, Rigoni R, Colleoni F, et al. Therapeutic Potential of Immunoproteasome Inhibition in Duchenne Muscular Dystrophy. Mol Ther, 24(11), 1898-1912. doi:10.1038/mt.2016.162.

6. Bello L, Pegoraro E. The "Usual Suspects": Genes for Inflamma-tion, Fibrosis, Regeneration, and Muscle Strength Modify DuchenneMuscular Dystrophy. J Clin Med, 8(5). doi:10.3390/jcm8050649.

7. Villalta SA, Rosenberg AS, \& Bluestone, J A. The immune system in Duchenne muscular dystrophy: Friend or foe. Rare Dis, 3(1), e1010966. doi:10.1080/21675511.2015.1010966 
8. Rosenberg AS, Puig M, Nagaraju K, Hoffman EP, Villalta SA, Rao V, et al. Immune-mediated pathology in Duchenne muscular dystrophy. Sci Transl Med, 7(299), 299 rv294.

doi:10.1126/scitransImed.aaa7322.

9. Donovan JM, Zimmer M, Offman E, Grant T, \& Jirousek M. A Novel NF-kappaB Inhibitor, Edasalonexent (CAT-1004), in Develop-ment as a Disease-Modifying Treatment for Patients With Duchenne Muscular Dystrophy: Phase 1 Safety, Pharmacokinetics, and Pharmacodynamics in Adult Subjects. J Clin Pharmacol, 57(5), 627-639. doi:10.1002/jcph.842.

10. Heredia JE,Mukundan L,Chen FM,Mueller AA, Deo RC, Locks-ley RM, et al.Type 2 innate signals stimulate fibro/adipogenic progenitors to facilitate muscle regeneration. Cell,153(3).,376388,2013.doi:10.1016/j.cel.2013.02.053.

11. Tidball JG, Welc SS, Wehling-Henricks M.Immunobiology of i-nherited Muscular Dystrophies.Compr Physiol.8(4):1313-1356. doi:10.1002/cphy.c170052.

12. Moreira A, Leisgang W, Schuler G, Heinzerling L. Eosinophilic count as a biomarker for prognosis of melanoma patients and its importance in the Response to immunotherapy. Immunotherapy.9(2)115121.doi:10.2217/imt-2016-0138.

13. Fuschillo S, Molino A, Stellato C, Motta A, Maniscalco M. Blood eosinophils as biomarkers of therapeutic response to chronic obstructive pulmonary disease: still work in progress. Eur Intern Med,2019,68;1-5 doi:10.1016/j.ejim.2019.07.005.

14. Okauchi S, Shiozawa T, Miyazaki K, Nishino K, Sasatani Y, O-hara G, et al.Association between peripheral eosinophils and clinicaloutcomes in patients with non-small cell lung cancer treated with $\mathrm{i}$ mmune checkpoint inhibitors.Pol Arch Intern Med .2012,131(2):152-160.doi:10.20452/pamv.15776.

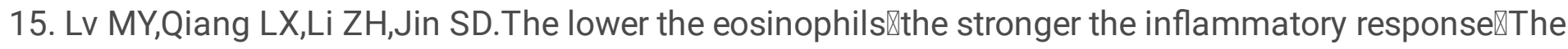
relationship of different le-vels of eosinophils with the degree of inflammation in acute exacerbation chronic obstructive pulmonary disease $\triangle A E C O P D \bigotimes$.Journal of Thoracic Disease,2021,13(1):232243.doi:10.21037/jtd-20-2178.

16. Ondari E, Calvino-Sanles E, First NJ, Gestal MC.Eosinphils andbacteria囚the beginning of a story.Int J Mol Sci.2021,22(15):8004.do-i囚10.3390/ijms22158004.

17. Schröder T, Fuchss J, Schneider I, Stoltenburg-Didinger G, Hani-sch F. Eosinophils in hereditary and inflammatory myopathies.Acta Myologica.2013,32(3):148-153.PMID: 24803842.

18. Sek AC,Moore IN,Smelkinson MG, Pak K, Minai M, Smith R, et al. Eosinophils do not drive a acute muscle pathology in the m-dx mouse model of Duchenne muscular dystrophy. J Immunol. 2019July 15; 203(2): 476-484. doi:10.4049/jimmunol.1900307.

19. Lu M, Taylor BV, Körner H. Genomic effects of the vitamin DReceptor: potentially the link between vitamin D,immune cells, and multiple sclerosis.Front Immunol.2018,12;9:477.doi:10.3389/fimmu.2018.00477eCollection 2018.

20. Mailhot G,White JH.Vitamin D and immunity in infants and children.Nutrients.2020,12(5):1233.doi:10.3390/nu12051233. 
21. Soslow JH, Markham LW, Burnette WB, Galindo CL, Feoktistov I,Raucci FJ, et al. Increased Number of Circulating CD8/CD26 T Cells in the Blood of Duchenne Muscular Dystrophy Patients Is as-sociated with Augmented Binding of Adenosine Deaminase and Higher Muscular Strength Scores. Frontiers in Pharmacology, 8. doi:10.3389/fphar.2017.00914.

22. Pinto-Mariz F, Rodrigues Carvalho L, Prufer De Queiroz Campos,Araujo A, De Mello W, Gonçalves Ribeiro $\mathrm{M}$, et al. CD49d is a disease progression biomarker and a potential target for immunothe-rapy in Duchenne muscular dystrophy. Skeletal Mus-cle, 5(1). doi:10.1186/s13395-015-0066-2.

23. Kharraz Y, Guerra J, Mann CJ, Serrano AL, Muñoz-Cánoves P. Macrophage plasticity and the role of inflammation in skeletal mus-cle repair. Mediators Inflamm, 2013, 491497. doi:10.1155/2013/491497.

24. Mojumdar K,Liang F, Giordano C, Lemaire C, Danialou G, O-kazaki T, et al.Inflammatory monocytes promote progression of Du-chenne muscular dystrophy and can be therapeutically targeted via CCR2. EMBO Mol Med, 6(11), 1476-1492. doi:10.15252/emmm.201403967.

25. McDonald CM, Sajeev G, Yao Z, McDonnell E, Elfring G, Sou-za M, et al. Deflazacort vs prednisone treatment for Duchenne muscular dystrophy: A meta-analysis of disease progression rates in recent multicenter clinical trials. Muscle Nerve, 61(1), 26-35. doi:10.1002/mus.26736.

26. Flanigan KM, Campbell K, Viollet L, Wang W, Gomez AM, W-alker, CM, et al.Anti-Dystrophin T Cell Responses in Duchenne M-uscular Dystrophy: Prevalence and a Glucocorticoid Treatment Effect.Human Gene Therapy, 24(9), 797-806. doi:10.1089/hum.2013.092.

27. Kameyama T, Ohuchi K, Funato M, Ando S, Inagaki S, Sato A.et al. Efficacy of Prednisolone in Generated Myotubes Derived Fr-om Fibroblasts of Duchenne Muscular Dystrophy Patients. Front Pharmacol, 9, 1402. doi:10.3389/fphar.2018.01402.

28. Herbelet S, Rodenbach A, Paepe B, De Bleecker JL. Anti-Infla-mmatory and General Glucocorticoid Physiology in Skeletal Muscles Affected by Duchenne Muscular Dystrophy: Exploration of SteroidSparing Agents. Int J Mol Sci, 21(13). doi:10.3390/ijms21134596.

\section{Figures}


Effects of different eosinophils levels on the efficacy of DMD

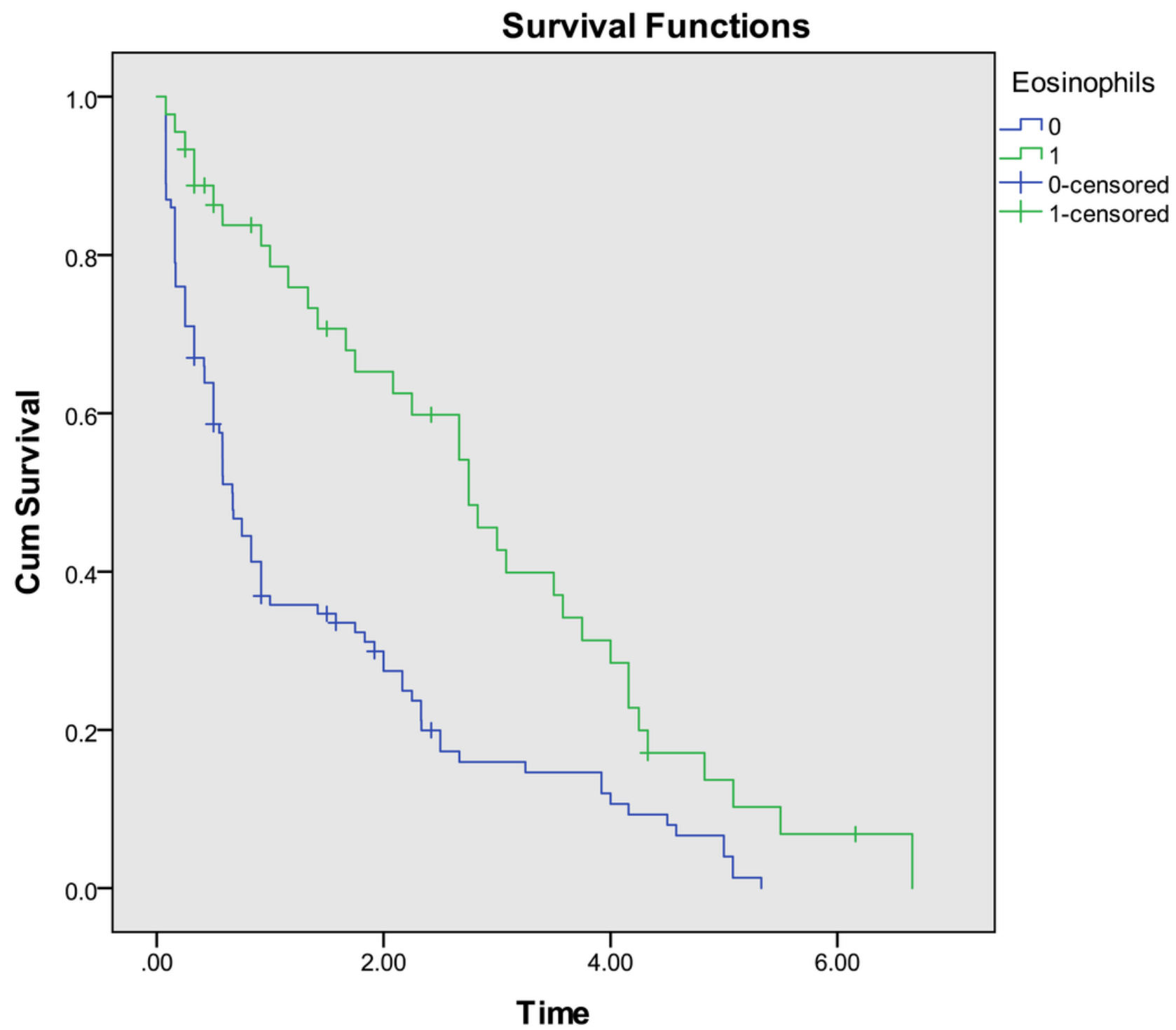

Log Rank 16.201, $\mathrm{P}=0.000$.The median survival time of eosinophils 0 level was 0.667 years, the standard deviation was 0.082 ; 1 level was 2.75 years, and the standard deviation was 0.359 .

\section{Figure 1}

Effects of different eosinophil levels on the efficacy of DMD 
Effects of different treatment methods on the efficacy of DMD

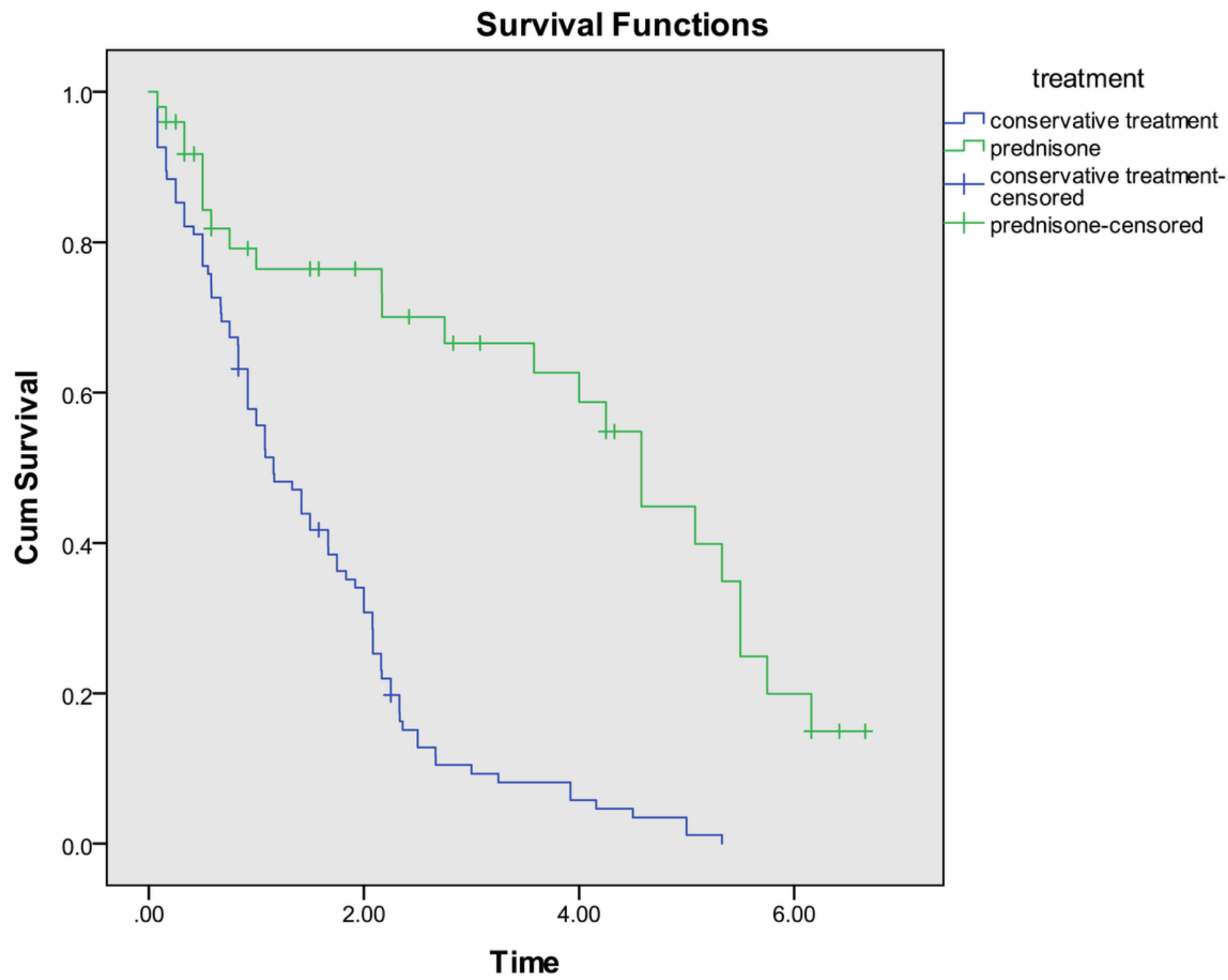

Log Rank 38.051, $\mathrm{P}=0.000$. The median survival time of the conservative treatment group was 1.160 years, and the standard deviation was 0.184 ; the prednisone treatment group was 4.580 years, and the standard deviation was 0.405 .

\section{Figure 2}

Effects of different treatment methods on the efficacy of DMD 
Effects of different eosinophils levels on the efficacy of DMD

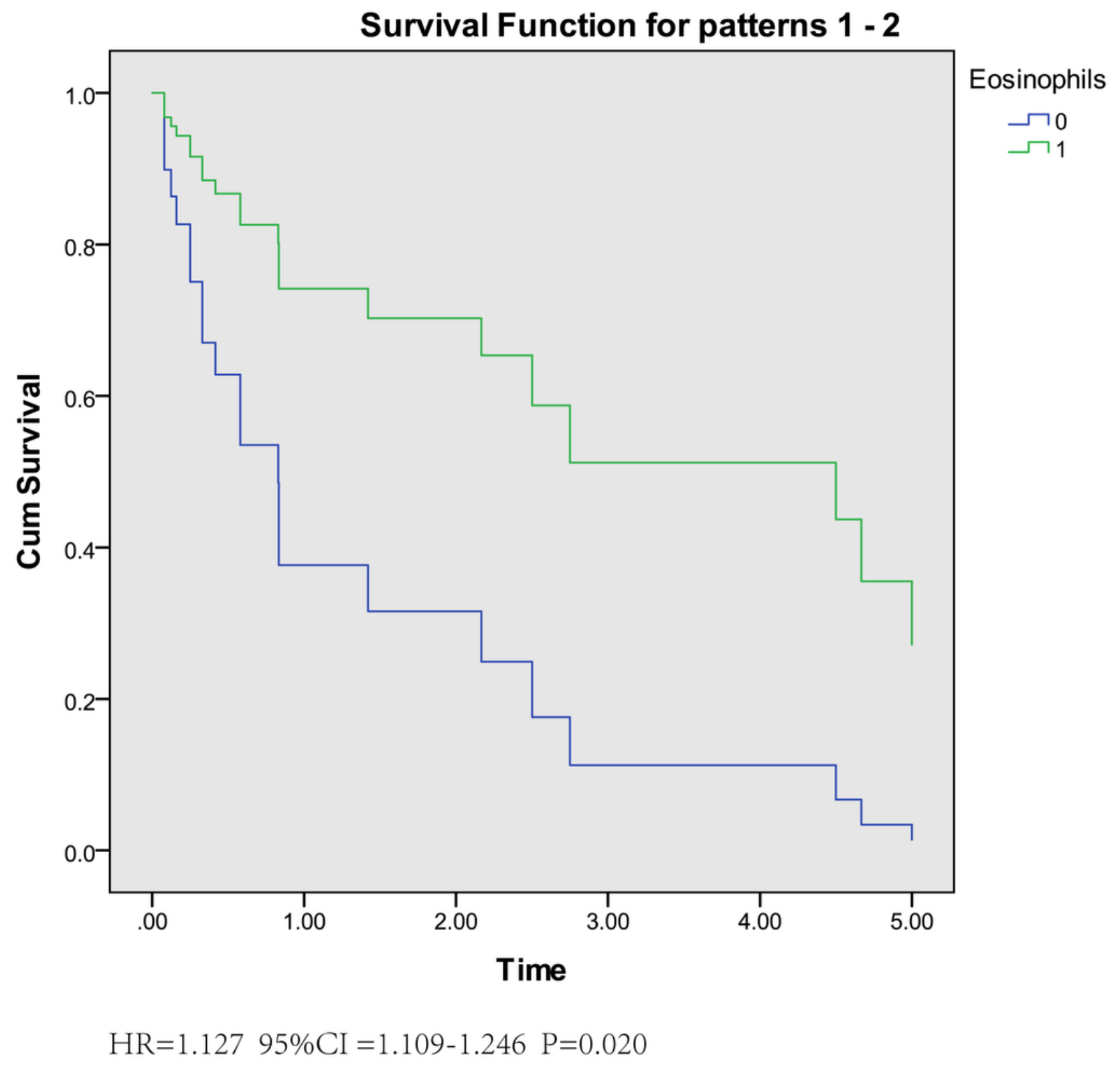

Figure 3

Effects of different eosinophil levels on the efficacy of DMD 